\title{
Experimental Hepatic Fibrosis Due to Capillaria hepatica Infection (Differential Features Presented by Rats and Mice)
}

\author{
Stelamares Boyda de Andrade, Zilton A Andrade ${ }^{+}$
}

\author{
Centro de Pesquisas Gonçalo Moniz-Fiocruz, Rua Valdemar Falcão 121, 40295-001 Salvador, BA, Brasil
}

\begin{abstract}
Rats and mice are among the most susceptible hosts for the helminth Capillaria hepatica. More information on the similarities and differences between the hepatic pathology presented by these two parasite hosts are needed, since they may represent good models for the study of hepatic fibrosis. Early changes are similar for both hosts and are represented by necro-inflammatory lesions around dead parasites and their eggs and diffuse and intense reactive hepatitis. Although worms remain alive longer in mice than in rats, hepatic changes are more rapidly and deeply modulated in the former, even leading to almost complete disappearance of fibrosis. As for the rats, the modulation of the focal lesions is followed by the formation of septal fibrosis, a process where fine and long fibrous septa appear connecting portal spaces and central veins in such a way as to form a final morphologic picture of cirrhosis. Hepatic functional changes usually present good correlations with the morphologic findings at the different phases of the infection evolution. Therefore $\mathrm{C}$. hepatica infection in rats and mice represent two different models of hepatic fibrosis and these differences, if properly known and understood, can be explored to answer different questions regarding several aspects of hepatic fibrosis
\end{abstract}

Key words: Capillaria hepatica - septal fibrosis - hepatic fibrosis - pathogenesis

Progressive hepatic fibrosis and cirrhosis constitute the most serious consequence of many chronic diseases affecting the liver. Considerable progress has recently occurred in our understanding of hepatic fibrosis. However, not only its pathogenesis and the cellular and molecular factors involved, but several other problems, especially related to reversibility and significance of different morphological types of fibrosis, still require further investigation (Friedman 1993, 2000, 2003, Bhunchet et al. 1996, Brown 2000, Poli 2000). Many of these problems can be experimentally tackled by resorting to one or more animal models available, as reviewed by Tsukamoto et al. (1990). The diversity of models is important because they represent different types and pathways of fibrosis development, and also the fibrogenic responses to different etiologies, each model contributing with their peculiarities to clarify especial details of a complex problem.

A new model of hepatic fibrosis was described in rats infected with the nematode Capillaria hepatica by Ferreira and Andrade (1993). Besides the focal necro-inflammatory parasitic lesions, caused by groups of worms that usually die and disintegrate within the liver soon after egg lying, there also occurs the development of septal fibrosis throughout the liver, a change that appears in all infected rats (Oliveira \& Andrade 2001). The model has permitted studies on pathogenesis (Santos et al. 2001, Lemos et al. 2003), on response to drugs (Souza et al.

Financial support: Papes III-Fiocruz

${ }^{+}$Corresponding author. Fax: +55-71-365.2155. E-mail: zilton@cpqgm.fiocruz.br

Received 22 January 2004

Accepted 2 June 2004
2000, 2001) and on other aspects (Ferreira \& Andrade 1993, Oliveira \& Andrade 2001). However, the potential of the model is somewhat limited by the peculiarities connected with the experimental host - the rat - because of the difficulties related to the obtaining of commercial kits to study cytokines, chemokines, receptors, growth factors, cells and extra-cellular tissue markers, and so on. Inasmuch as it was also observed that the septal fibrosis, so characteristic of the rat infection, did not occur in the mouse (Gotardo et al. 2000). However, the pathology of capillariasis in the mouse also includes the presence of chronic inflammation and fibrosis and other features worth of attention as far as hepatic fibrosis is concerned.

The present investigation was planned as a comparative study of the hepatic pathology induced by $C$. hepatica in mice and rats. It was expected that time-sequence data regarding the occurrence of different morphological types of hepatic fibrosis and on its accumulation and degradation would contribute to the understanding of pathogenesis and to better characterize each one experimental model.

\section{MATERIALS AND METHODS}

Animals -Twenty male 3-month-old Wistar rats, weighing 100 to $200 \mathrm{~g}$, and 60 male Swiss mice, weighing 10 to 15 $\mathrm{g}$ were used. They were maintained at the Gonçalo Moniz Research Center-Fiocruz, Salvador, BA, in rooms with controlled temperature $\left(22 \pm 2^{\circ} \mathrm{C}\right)$ and humidity $(55 \pm 10 \%)$ and continuous air renovation, with free access to a commercial balanced diet and water. Rats, as well as mice, were kept in spacious metallic cages, 5 per cage.

Inoculum - Consisted of C. hepatica embryonated eggs, administered by gavage in the dose of approximately 600 eggs to a rat and 100 eggs to a mouse. The eggs were obtained from the livers of experimentally infected rats, around the 40th day after inoculation. The livers of the donors were washed in order to get rid of excess blood, 
homogenized in an electrical blender, followed by several turns of washing with tap water and decantation, until the liquid above the sediment was completely clear. The clean immature eggs were kept moisturized with $0.5 \%$ formalin solution in a Petri dish, at room temperature $\left(26-28^{\circ} \mathrm{C}\right)$, for 28-30 days, in order to embryonate. They were counted under the microscope, and the infecting dose suspended in $0.5 \mathrm{ml}$ of distilled water per each animal.

Experimental groups - 1st group: rats were maintained alive throughout the time of the experiment. Blood and liver tissue samples were collected pre-infection and at the 15th, 40th, and 70th days post-infection; 2nd group: mice were sacrificed, 5 at a time, before infection and at the 30th, 45th, and 90th day post infection.

These different time points for rats and mice were intended to represent the most characteristic periods of the acute, intermediate, and chronic phases of the infection, according to previous experience of this laboratory (Ferreira \& Andrade 1993, Gotardo et al. 2000).

Materials collected at pre-infection time served as normal controls. Besides, in rats each animal served as its proper control, since materials collected from the same animals at different experimental times were always compared.

General procedures - Blood was collected from rats by puncture of a tail vein. From mice the collect was made after severing of the brachial plexus during exsanguinations of the anesthetized animal, previous to sacrifice.

Liver biopsies were performed in rats under general anesthesia and aseptic conditions. A surgical incision was made in the abdominal mid-line to expose the liver. A fragment of approximately 0.25 to $0.30 \mathrm{~g}$ was tied out and removed. Recovery of the operated animals was uneventful.

The mouse liver was obtained at autopsy.

Anti-C. hepatica antibody detection - Sera taken from rats and mice, collected at the several experimental points mentioned above, were used. An ELISA test for detection of total Ig antibodies using a peroxidase-conjugated goat anti-rat IgG (Sigma) was used. The plates were sensitized with $10 \mu \mathrm{g} / \mathrm{ml} \mathrm{C}$. hepatica egg antigen diluted in carbonate buffer, $\mathrm{pH}$ 9.6. Lecture was made on a microplate reader Molecular devices-Thermomax spectrophotometer (Sunnyvale, CA, US) at $450 \mathrm{~nm}$ connected to a computer with MDS-Soft Max.

Histology - Fragments of the liver obtained by biopsy (rats) or autopsy (mice) were fixed in phosphate buffered (pH 7.2) $10 \%$ formalin and embedded in paraffin. Sections were routinely stained with hematoxylin and eosin, and with the picro-sirius method for collagen, Perls' method for iron, the PAS-Schiff staining with and without previous treatment with diastasis, and orcein for elastic tissue.

Immunofluorescence - Fragments of liver tissue were immediately placed in liquid nitrogen for a few minutes and them kept frozen at $-70^{\circ} \mathrm{C}$ in airtight boxes, until the moment they were sectioned in a cryostat at $-20^{\circ} \mathrm{C}$. The sections were submitted to an indirect immunofluorescence technique for the demonstration of collagen isotypes (I, III, IV), laminin, fibronectin, and actin. The specific anti-sera were anti-human polyclonal, obtained in rabbits and were a gift from Mme Sylviane Guerret (In- stitute Bio-Merrieux, Lyon, France). Anti- $\alpha$ actin antibodies were purchased from Sigma (St. Louis, MO, US).

They were used in dilution varying from 1:40 to 1:100. Details concerning their preparation and tests of specificity can be found elsewhere (Andrade \& Grimaud 1986, 1988). Secondary fluoresceinated anti rabbit-IgG was commercially obtained from Sigma.

Statistical analysis - The Kruskal-Wallis and the Dunn multiple comparison non-parametric tests were used to compare serological data obtained from different experimental groups, considering $\mathrm{P}<0.05$ as significant.

\section{RESULTS}

Acute stage - Around 15 days of infection for the rats, and 30 for mice, mature and immature $C$. hepatica worms were seen to disintegrate inside the liver and to liberate eggs. These materials appeared surrounded by a central area of necrosis, frequently invaded by polymophonuclear leukocytes, and a peripheral ring of fibro-cellular reaction, sometimes forming a dense fibrous capsule. Mononuclear leukocytes, with predominance of macrophages and a few giant cells appeared around the worms debris and eggs. Sooner or later, some foci of calcification were noted. Outside these focal parasite lesions, the liver presented moderate to dense portal cellular infiltration, with mononuclear cells and many eosinophils, mobilization of sinusoidal cells, and some foci of isolated-cell necrosis (Fig. 1A). These changes of reactive hepatitis were much more severe in mice, with areas of dissociation and necrosis of liver cords and considerable egg deposition (Fig. $2 \mathrm{~A}, \mathrm{~B})$.

Intermediate stage - This stage of repair occurred about the same time for both rats and mice (40-45 days after inoculation) and was characterized by the subsiding of the reactive hepatitis. In rats live worms disappeared from the focal leions, being replaced by partially calcified worm debris (Fig. 1A), and collections of immature eggs. A repair tissue formed by fibroblast and blood vessel proliferation and macrophage infiltration later replaced the necrotic areas, but packed collections of eggs were still present, although in the absence of worm debris, especially in mice. The signs of non-specific hepatitis seen during the acute stage, completely disappeared in rats, but left a variable degree of peri-sinusoidal fibrosis in mice (Fig. 2C, D). However, septal fibrosis formation was a constant and prominent change seen in rats, although absent in mice. The early septa were thin and cellular, and they connected portal spaces to neighboring portal spaces sometimes forming polyhedral figures with the central area occupied by central veins, mimicking the normal histology of the pig liver. The septa were formed by parallel collagen fibers, fusiform cells (fibroblasts) and blood vessels (Fig. 1C, D). Hepatic cells present at the vicinity of the septum sometimes showed apoptotic changes, eventually dropping out and being incorporated into the septal structure. Some of them were seen to contain PASpositive, diastase-resistant material, and a brownish, ironcontaining pigment.

Chronic stage - Sections taken from the liver, 70 days after infection of rats and 90 days post infection of mice, revealed scattered, isolated fibrotic and calcified residual 



Fig 1: changes induced by Capillaria hepatica infection in the liver of rats. A: acute stage: severe reactive hepatitis and necrotic reaction around parasitic debris and eggs (arrows). The other pictures are representative of the intermediary and chronic stages; B: a calcified area caused by the parasite death appears surrounded by a fibrous capsule (arrows). Septal fibrosis limits nodular areas of parenchyma; C-D: early, predominantly cellular, septa appear crossing the hepatic parenchyma (arrows); E-F: the presence of collagen demonstrates the extension of $C$. hepatica-induced septal fibrosis. All pictures taken at magnification X100. A to D: Hematoxylin \& Eosin; E and F: Picro-sirius red for collagen 

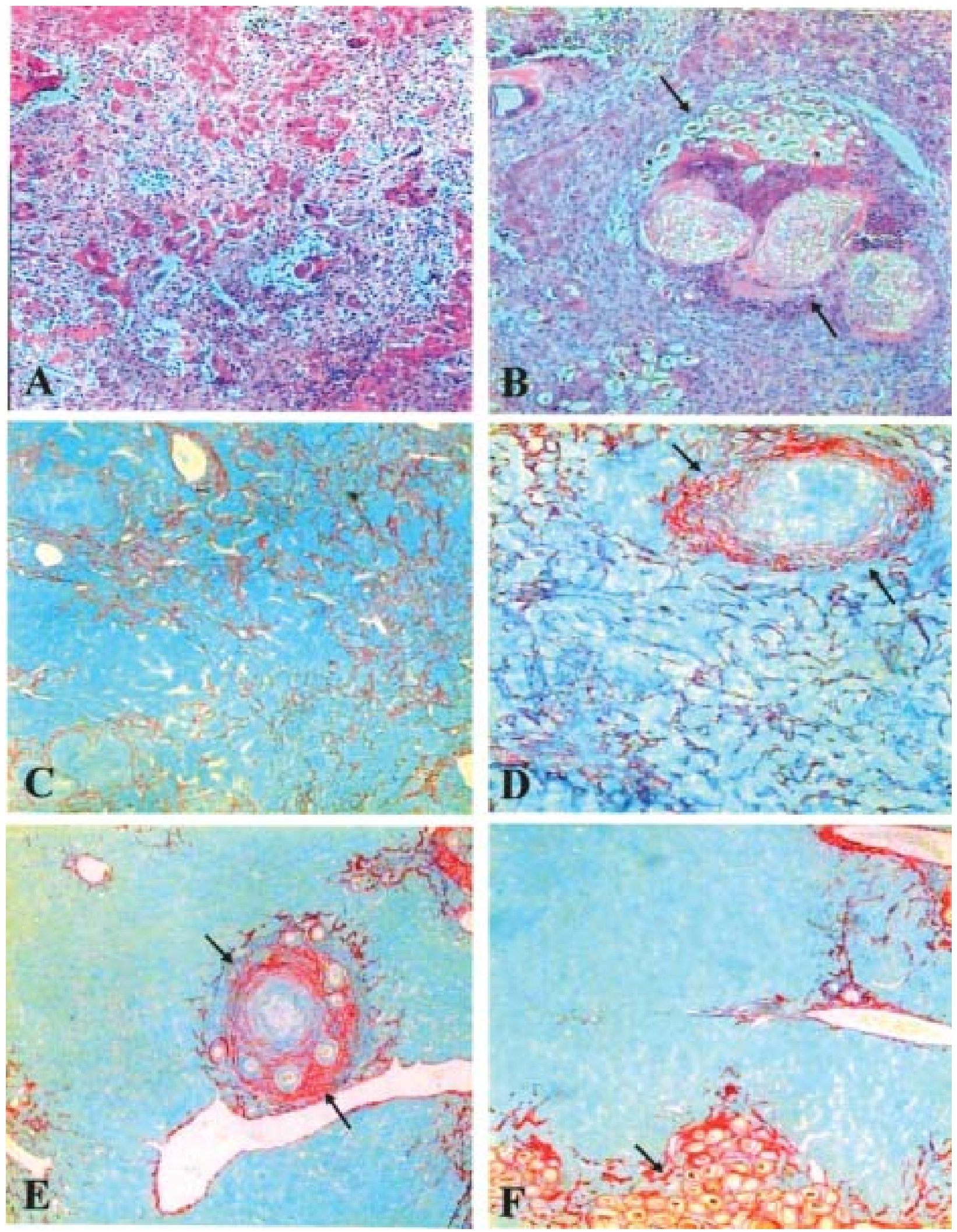

Fig 2: changes induced by Capillaria hepatica infection in the liver of mice. A-B: acute stage showing the severity of the necroinflammatory changes (A) and the large collections of eggs inside an encapsulated structure (B). Hematoxylin \& Eosin, X 100; C-D: intermediate stage. Diffuse perisinusoidal fibrosis dominates the picture. Picro-sirius red for collagen, C: X100; D: X200. E-F: chronic stage. Liver parenchyma is free of changes, except for the focal fibrosis around collections of parasite eggs. There are no traces of septal fibrosis. Picro-sirius red for collagen, X100 
parasitary lesions, containing clusters of eggs (Fig. 2E, F). However, aside from the focal fibrosis, the mouse liver histologically appeared to be within normal limits (Fig. 2E, F). Even within the fibrotic foci some degree of collagen degradation was observed in mice, with the collagen fibers becoming loosely arranged and frequently fragmented. Sometimes eggs were seen free among groups of liver cells. On the other hand, the septal fibrosis seen in rats during the previous stage, persisted. The cellular component of the septa almost disappeared and the fibrosis became more evident, as seen in sections stained with the picro-sirius method (Fig. 2E, F). Septal fibrosis was so marked in some focal areas as to dissociate nodular portions of the liver parenchyma, simulating a real cirrhosis, but usually the septa were long and appeared crisscrossing the parenchyma, separating large and irregular areas of the liver, forming a mosaic pattern.

Extra-cellular matrix changes - The septation of the liver parenchyma of rats and the fibrous capsules of the focal parasitic lesions were well evident when the sections were submitted to immunofluorescence to reveal the collagen isotypes, I, III, and IV, laminin and fibronectin (Fig. 3A, B, C). There was no differential behavior for fibronectin in the infected livers of rats and mice. Fibronectin appeared abundant in inflammatory lesions of the acute stage and gradually faded away subsequently. During the chronic stage a mild linear or granular staining for fibronectin was present wherever the connective tissue was present, similarly as seen in the normal control liver. By revealing basement membranes, laminin, as well as type IV collagen, appeared more prominent during the intermediate stage of the infection in rats, when septal fibrosis disclosed the presence of abundant blood vessels. These vessels were also exhibited when their muscular coats were stained positive for actin (Fig. 3D).

In mice, collagens I and III were present in areas of fibrosis from the beginning to the end of the experiment, but without any special connotation. The same for the other components of extra-cellular matrix searched (Fig. 3E, F).

Levels of serum anti-C. hepatica antibodies - Results are depicted in Fig. 4A for the rats and in Fig. 4B for mice. The situation was just the opposite for the two hosts. In rats the levels of antibodies gradually increased during the three experimental points, reaching their maximum at the end of the experimental period. In mice there was a considerable increase during the acute phase of the infection, with a moderate decrease at the intermediary stage and a negative test by the end of the experiment. Any result obtained between two experimental points was statistically significant $(\mathrm{P}<0.05)$.

\section{DISCUSSION}

Luttermoser (1938) made a comparative study of the infection caused in rats and mice by $C$. hepatica. That was a well-conducted study, mainly concerned with parasitology, but also containing important pathological data. He noted that the same type of gross lesions appeared about three days earlier in the rat infections in spite of the more rapid growth of the worms in the mice. Other than this difference in the rate of lesion change and the fact that all the mouse lesions became diffuse and egg-bearing, essentially the same gross pathological picture appeared in the mice as in the rats. He said that the slower reaction and faster parasitic growth did not allow the mouse to kill the worms before they matured and laid eggs, and therefore prevented the control of the infection which was found in the rat. In fact, all evidence of the injurious influence of massive $C$. hepatica infections was much more marked in the mouse than in the rat. The present study confirms the main lines of the Luttermoser's observations, but goes beyond that, observing that the mouse modulates faster and more completely the severe inflammation of the acute stage, and the fibrosis of the intermediate and chronic stages, when compared to the rat. Above all, it is impressive the extent and the rapidity of fibrosis resorption that occur in the mice. Such fibrosis is predominantly of the peri-sinusoidal type. This type is supposed to be particularly prone to degradation and resorption, as demonstrated by Lortat-Jacob et al. (1997) in experimental schistosomiasis under treatment. But, even if one considers the fibrosis that is part of the encapsulating process around dead worms and eggs, it also undergoes advanced degradation with time, to an effect that many eggs appear later on free within the hepatic parenchyma, as registered by Gotardo et al. (2000).

On the other hand, fibrosis in rats is quite peculiar. Fibrosis appearing around the focal parasitic lesions tends to remain for prolonged periods of time, at least six months (data not shown). When the worm debris already disappeared, and the eggs are reduced to a few, the fibrosis can still be seen encircling isolated calcified foci. But, the most peculiar aspect is the formation of septal fibrosis in rats infected with C. hepatica. It appears in 100\% of the infected animals, and it is evident regardless the parasite load (Oliveira \& Andrade 2001). Septal fibrosis extends from portal spaces, going toward neighboring portal spaces and central veins, finishing by involving the entire organ, thus creating a septate mosaic pattern (Ferreira \& Andrade 1993, Souza et al. 2000, 2001).

Septal fibrosis of the liver in C. hepatica-infected rats, although more marked, has the same distribution, and probably the same pathogenetic connotation (Lemos et al. 2003) of that induced in rats by repeated injections of whole pig serum or by its albumin fraction (Ballardini et al. 1983, Andrade 1991, Bhunchet et al. 1992, Paronetto \& Popper 1996). Actually, the C. hepatica and the pig-serum models are the two models of septal fibrosis being used in experimental investigations. C. hepatica-induced-septal fibrosis has been revealed as a good model for testing anti-fibrosis drugs and for studies on pathogenesis of hepatic fibrosis (Souza et al. 2000, 2001, Santos et al. 2001, Lemos et al. 2003).

On the other hand, the peculiarities of the hepatic fibrosis induced by $C$. hepatica infection in mice have been so far little explored. Little is known about the behavior of different genetic strains of mice in C. hepatica infection. A preliminary and unpublished study from our laboratory failed to disclose any evident differences when infection with $100 \mathrm{C}$. hepatica eggs was histologically compared in Swiss, Balb/c, C57, and AJ strains of mice. Besides the demonstration that mice, differently from rats, do not de- 

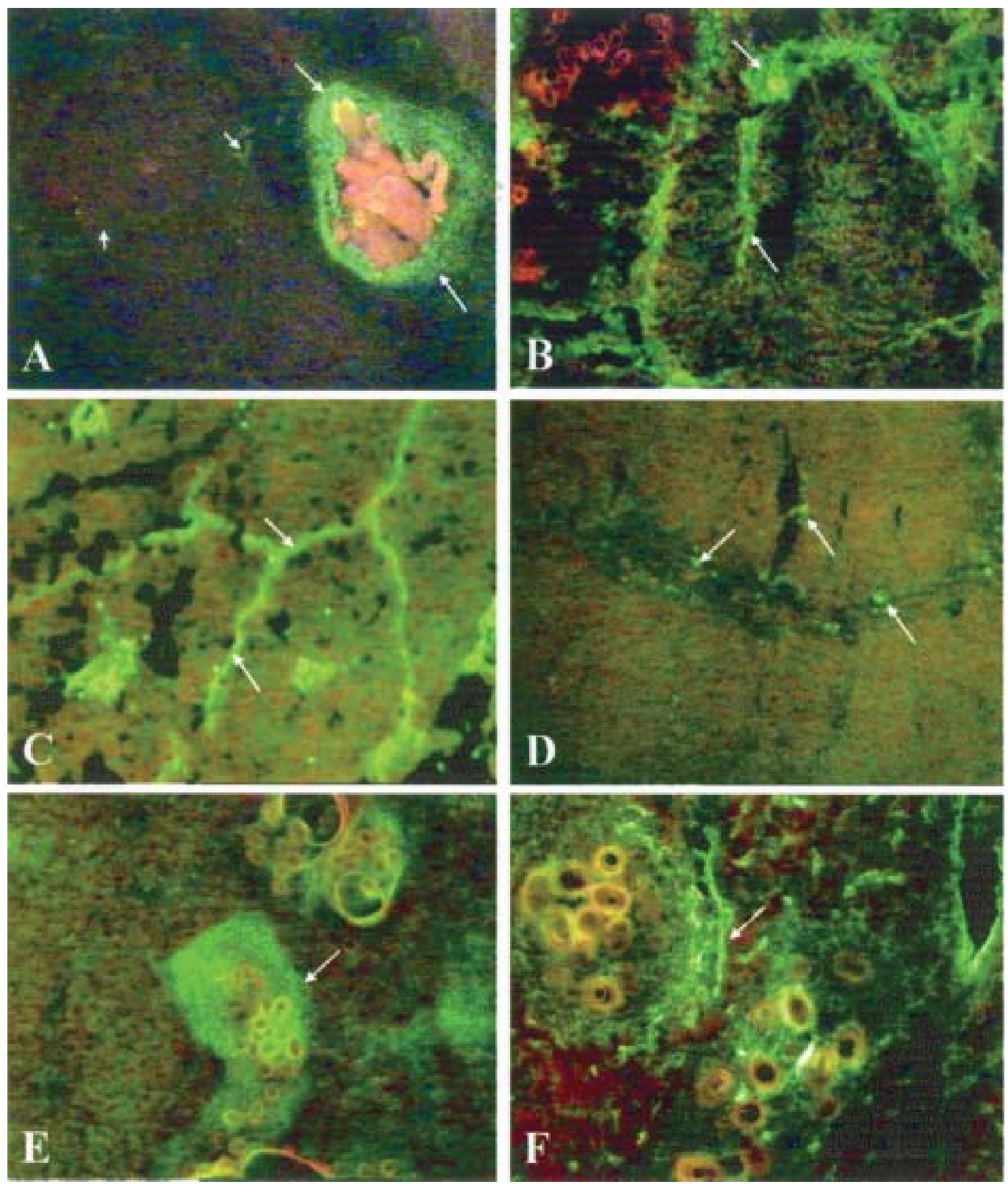

Fig. 3A to D: extra-cellular matrix changes observed during the infection of rats with Capillaria hepatica. A: Type I collagen is abundant in the fibrous tissues of the capsule formed around a collection of disintegrating parasites (long arrows), but is only weakly positive in the fibrous septa (short arrows) formed 40 days after infection; B: by the same time fibronecting is strongly positive in the septa (arrows). C: preparations for laminin also demonstrates bright fluorescence of the septa, especially by staining basement membrane of blood vessels (arrows); D: vessels within the septa can be seen when their muscular walls were staining for the presence of $\alpha$-actin (arrows); E-F: extracellular matrix changes observed during the infection of mice with $C$. hepatica. Presence of fibronectin (E) and laminin (F) in areas of periovular fibrosis at the chronic stage of the infection. Laminin marks the blood vessel basement membrane. All the pictures above were taken at magnification X200. 

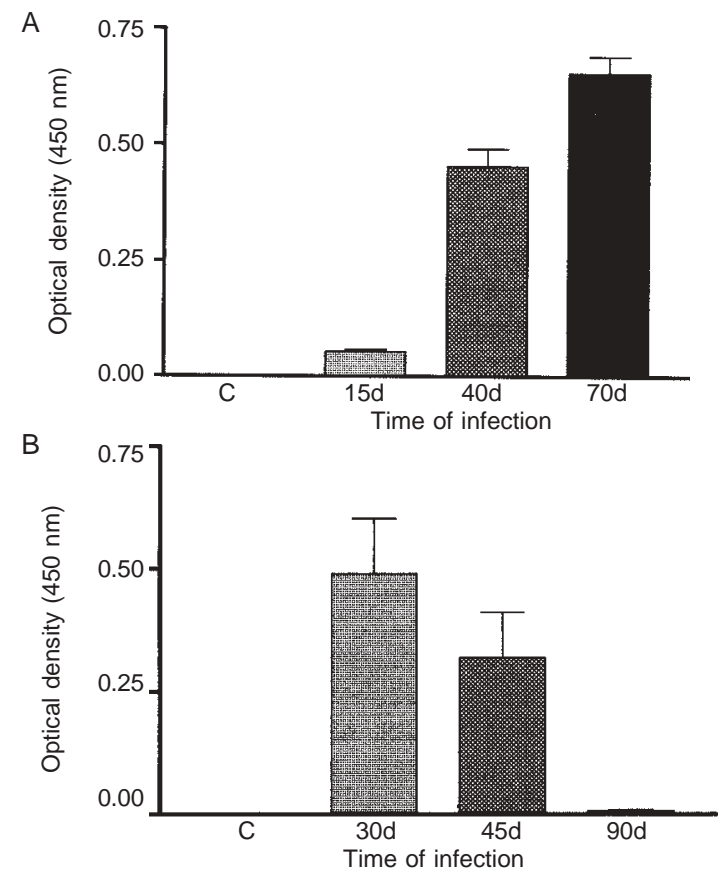

Fig 4A: anti-Capillaria hepatica antibody levels during different periods of the infection of rats; $\mathrm{P}<0.05$; $\mathrm{B}$ : anti-C. hepatica antibody levels during different periods of the infection of mice; $\mathrm{C}$ : control sera from intact animals; $\mathrm{P}<0.05$

velop septal fibrosis (Gotardo et al. 2000), no further studies are apparently on record. Since $C$. hepatica-induced fibrosis in mice disappears so rapidly and expontaneously, it may turn to be an adequate model for studies on the factors and mechanisms involved in extra-cellular matrix breakdown. Probably, it can be compared with other similar models such as that of the carrageenin granuloma (Perez-Tamayo 1970), the involution of the rat pregnant uterus (Henel1 et al. 1983), the periovular granuloma of schistosomiais (Andrade \& Grimaud 1986, 1988), and so on.

Immunological mechanisms may be at the roots of the differences in fibrosis production and degradation in $C$. hepatica-infected rats and mice, as suggested in a previous publication (Lemos et al. 2003). A striking difference was noted in the curve of the serum antibodies levels between the two hosts here considered. However, further studies are needed, especially considering the intrinsic cellular component of the hepatic immunological response, before one can draw some conclusions regarding the role of immunological factors to explain the differences found in rats and mice.

The main aim of this article is to call attention to the experimental models of hepatic fibrosis resulting from $C$. hepatica infection in two of its most frequent hosts. Both hosts respond with hepatic fibrosis of different morphological types and significance, and run different courses. It is expected that these differential features can be explored to answer questions on pathogenesis in relation to genetic and immunological background.

\section{REFERENCES}

Andrade ZA 1991. Contribution to the study of septal fibrosis of the liver. Internat J Exper Pathol 72: 553-562.

Andrade ZA Grimaud JA 1986. Evolution of the schistosomal hepatic lesions in mice after curative chemotherapy. Am J Pathol 124: 59-65.

Andrade ZA, Grimaud JA 1988. Morphology of chronic collagen resorption. (A study on the late stages of schistosomal granuloma involution). Am J Pathol 132: 389-399.

Ballardini G, Esposti SD, Bianchi FB, de Giolgi LB, Faccani A, Biolchini L, Busachi CA, Pisi E 1983. Correlation betwen Ito cells and fibrogenesis in an experimental model of hepatic fibrosis: a sequential stereological study. Liver 3: 5863.

Bhunchet E, Wake K 1992. Role of mesenchymal cell populations in porcine serum-induced rat liver fibrosis. Hepatology 16: 1452-1473.

Bhunchet E, Eishi Y, Wake K 1996. Contribution of immune response to the hepatic fibrosis induced by porcine serum. Hepatology 23: 381-387.

Brown KE 2000. An overview of hepatic fibrogenesis. Viral Hepatitis Rev 6: 5-27.

Ferreira LA, Andrade ZA 1993. Capillaria hepatica: a cause of septal fibrosis of the liver. Mem Inst Oswaldo Cruz 88: 441-447.

Friedman SL 1993. The cellular basis of hepatic fibrosis: mechanisms and treatment strategies. New Engl J Med 328: 18281835.

Friedman SL 2000. Molecular regulation of hepatic fibrosis, an integrated cellular response to tissue injury. $\mathrm{J} \mathrm{Biol} \mathrm{Chem}$ 275: 2247-2250.

Friedman SL 2003. Liver fibrosis - From bench to bedside. $J$ Hepatol 38: 38-53.

Gotardo BM, Andrade RG, Andrade ZA 2000. Hepatic pathology in Capillaria hepatica infected mice. Rev Soc Bras Med Trop 34: 341-346.

Henell F, Bricsson JLE, Glaumann H 1983. An electron microscopic study of the post partum involution of the rat uterus: with a note on apparent crinophagy of collagen. Virchows Arch B, Cell Pathol 42: 271-287.

Lemos QT, Magalhães-Fernandes I, Andrade ZA 2003. Immunological basis of septal fibrosis of the liver in Capillaria hepatica-infected rats. Braz J Med Biol Res 36: 1201-1207.

Lortat-Jacob H, Baltzer F, Desmouliere A, Peyrol S, Grimaud JA 1997. Lobular - but not periovular inhibition of collagen deposition in the liver of $S$. mansoni-infected mice using interferon-g. J Hepatol 26: 894-903.

Luttermoser GW 1938. An experimental study of Capillaria hepatica in the rat and the mouse. Am J Trop Med Hyg 27: 321-340.

Oliveira RF, Andrade ZA 2001. Worm load and septal fibrosis of the liver in Capillaria hepatica-infected rats. Mem Inst Oswaldo Cruz 96: 1001-1003.

Paronetto F, Popper H 1966. Chronic liver injury induced by immunologic reactions. Cirrhosis following immunization with heterologous sera. Am J Pathol 40: 1087-101.

Perez-Tamayo R 1970. Collagen resorption in carrageenin granulomas. II. Ultrastructure of collagen resorption. Lab Invest 22: 142-157.

Poli G 2000. Pathogenesis of liver fibrosis: role of oxidative stress. Mol Aspects Med 21: 49-98.

Santos AB, Tolentino Jr M, Andrade ZA 2001. Pathogenesis of hepatic septal fibrosis associated with Capillaria hepatica infection of rats. Rev Soc Bras Med Trop 34: 503-506.

Souza MM, Paraná R, Trepo C, Barbosa Jr AA, Oliveira I, 
Andrade ZA 2001. Effect of interferon- $\alpha$ on experimental septal fibrosis of the liver - Study with a new model. Mem Inst Oswaldo Cruz 96: 343-348.

Souza MM, Silva LM, Barbosa Jr A, Oliveira IR, Paraná R, Andrade ZA 2000. Hepatic capillariasis in rats: a new model for testing anti-fibrosis drugs. Braz J Med Biol Res 33: 13291334.

Tsukamoto H, Matsuoka M, French SW 1990. Experimental models of hepatic fibrosis. A Review. Sem Liver Diseases 10: $56-65$ 